\title{
Erratum to: Exclusive photoproduction of a heavy vector meson in QCD
}

\author{
D. Yu. Ivanov ${ }^{1, a}$, A. Schäfer ${ }^{2}$, L. Szymanowski ${ }^{3}$, G. Krasnikov ${ }^{2,4}$ \\ ${ }^{1}$ Institute of Mathematics, 630090 Novosibirsk, Russia \\ ${ }^{2}$ Institut für Theoretische Physik, Universität Regensburg, 93040 Regensburg, Germany \\ ${ }^{3}$ Soltan Institute for Nuclear Studies, Hoza 69, 00-681 Warsaw, Poland \\ ${ }^{4}$ Department of Theoretical Physics, St. Petersburg State University, 198904 St. Petersburg, Russia
}

Received: 15 January 2015 / Accepted: 28 January 2015 / Published online: 17 February 2015

(C) The Author(s) 2015. This article is published with open access at Springerlink.com

\section{Erratum to: Eur. Phys. J. C 34, 297-316 (2004) DOI 10.1140/epjc/s2004-01712-x}

The Eq. (2.13) contains an error related to improper counting of number of transverse dimensions within the dimensional regularisation method with the dimension $d=4+2 \epsilon=$ $2+2(1+\epsilon)$. The correct Eq. (2.13) is obtained with the help of substitution:

$$
\left(\frac{-g_{\mu \nu}^{\perp}}{2}\right) \rightarrow\left(\frac{-g_{\mu \nu}^{\perp}}{2(1+\epsilon)}\right)
$$

that can be understood as making an average over the number of transverse polarization states available to the gluons in $d$-dimensions, see also Eq. (10) in [1]. Note that this prescription is in accordance with conventional definition of the evolution kernels needed also for the subtraction of collinear divergences. We are grateful to Kornelija Passek-Kumericki and Dieter Müller for the discussion of these issues.

This correction requires the following changes in some intermediate results:

1. The corrected expression for $T_{g}(x, \xi)$ in the first line of Eq. (2.16) is obtained after the substitution in (2.16)

$$
\begin{aligned}
& \frac{\xi}{(x-\xi+i \varepsilon)(x+\xi-i \varepsilon)} \\
& \rightarrow \quad \frac{\xi}{(x-\xi+i \varepsilon)(x+\xi-i \varepsilon)(1+\epsilon)}
\end{aligned}
$$

The online version of the original article can be found under doi:10.1140/epjc/s2004-01712-x.

a e-mail: d-ivanov@math.nsc.ru
2. The corrected expressions for $\tilde{T}_{g}^{(0)}(x, \xi)$ and $\tilde{T}_{g}^{(1)}(x, \xi)$ in Eq. (3.56) are obtained after the substitution in (3.56)

$$
\frac{\xi}{(x-\xi)(x+\xi)} \rightarrow \frac{\xi}{(x-\xi)(x+\xi)(1+\epsilon)}
$$

3. The corrected expressions for the gluonic counterterm $\Delta_{g}^{\text {coll }}(x, \xi)$ in Eq. (3.62) and for the quark counterterm $\Delta_{q}^{\text {coll }}(x, \xi)$ in Eq. (3.63) are obtained after the substitutions in Eqs. (3.62) and (3.63)

$$
\frac{1}{\hat{\epsilon}}+1+\ln \left(\frac{\mu_{\mathrm{F}}^{2}}{\mu^{2}}\right) \rightarrow \frac{1}{\hat{\epsilon}}+\ln \left(\frac{\mu_{\mathrm{F}}^{2}}{\mu^{2}}\right)
$$

4. The corrected expression for the renormalisation of the strong coupling constant counterterm $\Delta_{g}^{\alpha_{\mathrm{S}}}(x, \xi)$ in Eq. (3.67) is obtained after the substitution in (3.67)

$$
\frac{1}{\hat{\epsilon}}+1+\ln \left(\frac{\mu_{\mathrm{R}}^{2}}{\mu^{2}}\right) \rightarrow \frac{1}{\hat{\epsilon}}+\ln \left(\frac{\mu_{\mathrm{R}}^{2}}{\mu^{2}}\right) .
$$

The final expressions for the gluonic hard scattering amplitude $T_{g}(x, \xi)$ of Eqs. (3.72) and (3.73) remain unchanged. Although the corrections in intermediate steps listed above affect all terms defining the finite part of $T_{g}(x, \xi)$ in the first line of Eq. (3.69), the net effect on the final result is zero.

The final expressions for the quark hard scattering amplitude $T_{q}(x, \xi)$ of Eqs. (3.70) and (3.71) has to be changed due to the correction of the quark collinear counterterm (3.63). The corrected form of Eq. (3.71) is obtained after performing the substitution

$\ln \frac{4 m^{2}}{\mu_{\mathrm{F}}^{2}}-1 \rightarrow \ln \frac{4 m^{2}}{\mu_{\mathrm{F}}^{2}}$

in the second line of Eq. (3.71). 
The above corrections do not change the main conclusions of the paper based on the numerical analysis performed.

Open Access This article is distributed under the terms of the Creative Commons Attribution License which permits any use, distribution, and reproduction in any medium, provided the original author(s) and the source are credited.

Funded by $\mathrm{SCOAP}^{3}$ / License Version CC BY 4.0.

\section{Reference}

1. L. Mankiewicz, G. Piller, E. Stein, M. Vanttinen, T. Weigl, Phys. Lett. B 425, 186 (1998) 